\title{
Three-Dimensional Computed Tomography for Planning Urologic Surgery
}

\section{Citation}

Young, Geoffrey S., Stuart G. Silverman, Joachim Kettenbach, Nobuhiko Hata, Polina Golland, Ferenc A. Jolesz, Kevin R. Loughlin, and Ron Kikinis. 1998. "THREE-DIMENSIONAL COMPUTED TOMOGRAPHY FOR PLANNING UROLOGIC SURGERY." Urologic Clinics of North America 25 (1) (February): 103-111. doi:10.1016/s0094-0143(05)70437-7.

\section{Published Version}

doi:10.1016/S0094-0143(05)70437-7

\section{Permanent link}

http://nrs.harvard.edu/urn-3:HUL.InstRepos:33956871

\section{Terms of Use}

This article was downloaded from Harvard University's DASH repository, and is made available under the terms and conditions applicable to Other Posted Material, as set forth at http:// nrs.harvard.edu/urn-3:HUL.InstRepos:dash.current.terms-of-use\#LAA

\section{Share Your Story}

The Harvard community has made this article openly available.

Please share how this access benefits you. Submit a story.

\section{Accessibility}




\title{
THREE-DIMENSIONAL CT FOR PLANNING UROLOGIC SURGERY
}

\author{
Geoffrey S. Young ${ }^{1}$, Stuart G. Silverman ${ }^{1}$, Joachim Kettenbach ${ }^{1}$, Nobuhiko Hata ${ }^{1}$, Polina Golland ${ }^{3}$, \\ Ferenc A. Jolesz ${ }^{1}$, Kevin R. Loughlin ${ }^{2}$, Ron Kikinis ${ }^{1}$
}

${ }^{1}$ Department of Radiology, Brigham and Women Hospital and Harvard Medical School

2 Department of Surgery, Brigham and Women's Hospital and Harvard Medical School

${ }^{3}$ Artificial Intelligence Laboratory, Massachusetts Institute of Technology, Cambridge, MA.

Acknowledgments: The authors wish to acknowledge the following grants NIH PO1 CA 67165-01A1 (R.K., FAJ), PO1 AG04953-14 (R.K.), NSF BES-9631710 (R.K.), DARPA F41624-96-2-0001 (R.K. and F.A.J.) and Austrian Science Fund, Project Jo 1315-MED (J.K.), Japan Society for Promotion of Science, Tokyo, Japan (N.H.).

\section{Introduction}

The development and marketing of new volumetric CT scanners at the turn of the decade made it possible to perform three dimensional imaging of the abdomen without respiratory artifacts and with clarity similar to that achieved in the musculoskeletal and central nervous systems by conventional scanners (Kalendar WA et al. 1990). Prior to 1990, all CT scanners had x-ray tubes that were connected to the machine's gantry using electrical cables. This limited the excursion of the tube in any one direction because continuous rotation would wind the cables into a knot. Thus, conventional CT scanning is performed by executing a series of individual CT scan slices during which the patient is instructed to hold their breath. Between scans the table is moved forward a certain distance and the process is repeated. If the breath holds are not identical, the imaged organ or region contains gaps or skip areas. This limitation is termed "respiratory misregistration". The technical advantage that made volumetric CT possible was the development of a continuously rotating (CT) X-ray tube with slip-rings. Slip rings are a pair of matched rings on the tube and gantry that can rotate past one another without limit. This allows for continuous rotation of the tube and the ability to perform a continuous X-ray exposure as the patient is moved forward through the CT gantry. The resulting exposure forms a path that looks like a spiral or helix, in shape, hence today volumetric CT scanning is often referred to as "spiral" or "helical" CT. Spiral CT acquires data in a region of interest using a single continuous X-ray exposure that is fast enough to be executed during a single breath hold, as the patient moves through the gantry, so that respiratory misregistration is eliminated. An entire body region is imaged and a continuous volume of CT data obtained. The resulting volumetric CT data set can be used to create axial images of at desired slice thickness and at a desired increment. Standard axial images from spiral CT of the urinary tract has been shown to be helpful in the diagnosis and staging of renal masses and are now routinely used for evaluation of renal lesions (Silverman SG et al 1994 and 1996). However, the same spiral CT data can be reformated in multiple planes or in three dimensions.

Although newer 3D reconstruction techniques retain more data than older reformatted display methods, 3D images cannot contain more information than appropriately filmed axial images from the same source data set. Thus, with the possible exception of CT angiography and future developments in virtual endoscopy, 3D images, by themselves, are of limited diagnostic utility. However, 3D imaging of the urinary tract is very useful in surgical planning, because it allows surgeons to visualize 3D anatomic relationships clearly. 3D images created from the same spiral CT acquired for diagnosis and staging of renal masses have been used to create color-coded 3D surface renderings of renal masses and surrounding structures for surgical planning of partial nephrectomy (Chernoff et al, 1994). A preliminary investigation and one published case report (Madjar et al 1996) have shown that this technique can help the urologist locate small renal masses and can help delineate the relationship between a mass and the urinary collecting system during operative planning. Currently, in addition to standard spiral CT, conventional pre-operative assessment of a patient before a partial nephrectomy may include 
aortography and selective renal angiography to determine the number, location, and pattern of branching of renal vessels. Intravenous pyelography may also be indicated to evaluate the anatomic relationship of any renal masses to the intrarenal collecting system and proximal ureter. Someday, three-dimensional rendering of the same spiral CT data set used to diagnose the tumor may be able to display the relationship between the tumor and the renal parenchyma, vasculature and collecting system thus obviating the need for these additional tests..

\section{D Data Rendering Techniques}

The following methods have been used to produce images from volumetric CT data which display 3D relationships more effectively than standard axial images: multiplanar reconstruction, curved planar reformatting, maximum intensity projection, binary shaded surface display, multi-threshold shaded surface display, and volume rendering (Rubin et al 1995).

Multiplanar reconstruction may be performed using software included with most modern CT scanners. These software packages produce images in the conventional anatomic planes (axial, sagittal, coronal) and any other preselected planes. The planes can often be made thick enough to display the full longitudinal extent of a linear or curvilinear structure, but individual reconstructed images usually can not display the full length of structures that curve through several planes, such as ureters or vessels. (Fishman et al 1991 and Rubin et al 1995)

Curved planar reformatting involves specifying a curved plane within the 3D source dataset that follows the long axis of a curving structure such as a blood vessel or a ureter. The resulting images allow complete longitudinal visualization of a single curving structure, but do not allow simultaneous visualization of the complete longitudinal extent of more than one curvilinear structure. When using these images for surgical planning, it is critical to remember that the "curved plane", on which the resulting images are based, produces a non-Cartesian coordinate display, which distorts distances and anatomic relationships (Fishman et al 1991 and $\underline{\text { Rubin et al }}$ 1995).

Maximum intensity projections (MIP) are constructed by defining a single direction, mathematically projecting rays along this direction through the original spiral data set, and mapping the highest attenuation encountered by any individual ray onto a single plane perpendicular to the predefined direction. Data from the entire thickness of the patient's body is projected onto a single flat plane, thus providing good longitudinal visualization of long curving structures such as vessels and ureters without distorting distances and anatomic relationships. However, the projection of each image onto a single plane sacrifices the 3D information contained in the original spiral CT data set. The images are similar to those created by plain film radiography, fluoroscopy or angiography, in the sense that, while images can be produced from a number of different angles, the individual images contain no 3D information. Thus, multiple MIP images are required to visualize 3D relationships just as AP, lateral, and oblique views are used in plain film radiography. Usually, MIP images are created from different angles around the patient, or a number of MIP images are created which are viewed as a cine loop. The cine loop method improves three-dimensional visualization, but remains somewhat cumbersome for viewing, and does not provide an easily accessible 3D image for quick reference during surgery or surgical planning. Equally importantly, only high attenuation structures can be imaged reliably with MIP. Moreover, high attenuation regions within the source dataset, such as those representing metal, calcium, or intense focal enhancement, will mask lower attenuation structures of interest which project behind them. Such high attenuation regions may have to be removed from the dataset by manual or semi-automated editing techniques to allow the production of useful images. Thus, MIP images can be quite useful for displaying a CT angiogram because only highly enhanced vascular structures are of primary interest, and because the limitations posed by the cine viewing are similar to those encountered in the interpretation of a conventional angiogram (Figure 1). However, this technique does not provide easily comprehensible 3D display of the data acquired in standard abdominal or urologic CT imaging protocols, nor can it display the anatomic relationships necessary for optimal surgical planning. This is particularly true when multiple structures are of interest at the same time, as in planning of urologic surgery, because MIP imaages will display only the structure with the highest attenuation.

Shaded Surface Display (SSD) images are created by identifying structures of interest within the source data set on the basis of their CT attenuation values. This process is called "segmentation" and represents an entire subbranch of applied computer science. Following segmentation, mathematical surface models are constructed of 
defined objects. The models are then rendered as surfaces, illuminated by an imaginary light source. Each individual view contains information about 3D relationships encoded in the imaginary perspective created by the shaded surface. Furthermore, reliable sizes, distances, and anatomic relationships are depicted. These features convey obvious advantages for surgical planning compared with the previously described techniques or the axial source images. However, in the most readily produced SSD images, where a single attenuation threshold is used, the data set is converted from a continuous range of CT attenuation data to a binary data set with significant loss of information. Specifically, during the editing process, anatomic details inside or outside a surface are removed. Thus the intrarenal portion of tumor, calculi, vessels, or collecting system cannot be displayed at the same time as the surface of the renal cortex or tumor. Moreover, subtle attenuation differences between tumor and kidney can be displayed.

It is possible to create multi-threshold SSD (MSSD) images which preserve more data than the single threshold ("binary") SSD images. The attenuation differences between individual tissues are preserved, allowing each structure of interest in the dataset to be assigned an arbitrary color to set it apart from surrounding tissues and organs. The images generated from this dataset may be viewed on a video display and rotated at will, or filmed from any angle. Workstation class computers currently come equipped with the dedicated hardware needed to accelerate the rendering process, and this type of display probably will become available on standard personal computers within the near future. Although some amount of the continuous CT attenuation data in the original spiral CT dataset is inevitably lost, this technique allows production of images that adequately depict most of the data relevant to surgical planning. Thus MSSD has been the technique most extensively demonstrated in urologic surgical planning to date (Chernoff et al 1994). However, the segmentation process required to produce images with this level of sophistication and detail is very labor intensive, and requires manual editing.

Furthermore, the reliability and usefulness of the resultant images are quite dependent on the experience and efforts of the radiologist involved in image production. In the future, semi-automated segmentation techniques may reduce this time cost substantially, but fully automated MSSD production which would obviate the need for manual editing is unlikely to become available in the foreseeable future.

One final technique has been described (Sommer et al 1996): volume rendering. In volume rendering, a histogram of attenuation values is generated from a given volume of the spiral CT data set, and different attenuation values are assigned different colors, transparencies, and refractive indices (Rubin et al 1995). Volume rendering demands very expensive hardware and software. However, the resulting images display transparent, colored surfaces illuminated by an imaginary light source, similar to MSSD images, but at the same time also depict details of structures inside and outside of the displayed surfaces. Moreover, all of the subtle CT attenuation differences within the original spiral CT data set are preserved in the volume rendering output data set. The detailed internal structure of tissues within a displayed surface may be visualized by suitably altering the color, transparency, and refractive index assigned to each tissue at the time the image is created. Thus, the output dataset preserves all of the data in the original dataset as well as allowing display of the 3D conformation of surfaces and simultaneous depiction of the depth and internal structure of tissues, organs, tumors and calculi. The flexibility of the output display and the comprehensive nature of the output data set are obvious advantages of volume rendering. However, the large output data set requires more sophisticated hardware, higher processor speed, more RAM, higher software costs, and significantly more of the radiologist's time. At the current level of technology, these costs prohibit widespread application of volume rendering.

Future advances in hardware may ultimately make volume rendering the display mode of choice. However, it may not be necessary to preserve or simultaneously display all of the data within a spiral CT data set in order to facilitate surgical planning. The source axial images from a spiral CT scan retain most of the diagnostically useful data from the original source dataset and, in most cases, will be superior to any reconstructed data set for diagnostic purposes. Moreover, judicious choice of thresholding may allow the production of a limited set of MSSD images that provide the same clinical benefit in surgical planning as volume rendered images at a much smaller hardware and professional time cost. In fact, this editing process may prove to be an advantage for surgical planning. By simplifying the anatomic relationships within the original source dataset and removing unnecessary anatomic detail, the images may provide a more accessible surgical roadmap. Furthermore, as described later, it is possible to display the fine internal structure of organs or tissues with advanced varients of 
MSSD imaging by displaying the surface renderings superimposed on the source axial images. This technique should address one of the most important shortcomings of MSSD images.

In summary, there are several techniques available for the post-processing of abdominal spiral CT data. Currently, multiplanar, curved reformatting and MIP are readily available on most commercial systems, while MSSD and volume rendering techniques are typically limited to dedicated workstations. Volume rendering has potential advantages for diagnostic purposes, but requires expensive hardware. Production of MSSD images requires the radiologist to define boundaries between different structures before the images are produced, which may aid the surgeon using the images for operative planning. Also, the dedicated hardware it requires is significantly less expensive than that needed for volume rendering.

\section{Clinical Studies To Date}

Preliminary experience has been reported with the use of color coded MSSD images to plan partial nephrectomy (Chernoff et al 1994). Data from the post-contrast portion of the spiral CT protocol employed for the diagnostic evaluation of renal masses was used in the 3D reconstruction. The resulting surfaces were displayed using a gradient shading MSSD technique, representing each tissue by a different color. Rotations of the image were performed by the software in about $5 \mathrm{sec} / \mathrm{view}$ and the views stored. Later, the kidney and its surrounding structures were depicted in multiple planes and displayed in a movie loop in which structures were selectively "peeled" away to reveal key anatomic relationships. The operator was able to cut, rotate, and translate individual or multiple tissues in order to display the anatomy of overlapping surfaces. These techniques were applied in a small number of patients undergoing partial nephrectomy and were judged by urologists to be helpful in locating small tumors during resection of indeterminate renal masses. Furthermore, the procedure was found to be helpful in planning the complete resection of large tumors, so as to obtain clean surgical margins but spare as much normal parenchyma as possible.

The urologists participating in the study reported that the technique effectively displayed the size and location of renal tumors, and the relationship between the tumors and the normal renal parenchyma. Overall, this was felt to facilitate alterations to the operative plan which preserved renal parenchyma. However, urologists graded the technique as less effective in (1) displaying the correct lesion depth and intrarenal location, and (2) displaying the relationship between the tumor and the collecting system and vessels (Chernoff et al 1994). The deficiency in display of lesion depth and intrarenal location stems from an intrinsic limitation of MSSD images. As discussed, the depth and intrarenal extent of a tumor could not be displayed on the same image as the tumor surface using the MSSD techniques employed during the study. Advanced MSSD or volume rendering images could correct this. Ineffective display of the relationship between the tumor and the renal vessels and collecting system is a more significant limitation, and will have to be addressed if 3D images are to prove useful as a surgical planning aid (lson et al 1995).

As discussed, a tumor relationship to the intrarenal collecting system and vasculature cannot be displayed in a single MSSD image along with the renal parenchyma. Although this limitation could be addressed by more advanced data rendering techniques, effective display of vessels and collecting system will still be influenced by the limitations of the original spiral CT dataset. Spiral CT protocols have been designed for optimal diagnosis and characterization of renal masses, employing contrast enhanced images during the nephrographic phase (typically $100 \mathrm{sec}$ after contrast material is injected) (Silverman et al 1994). The collecting systems will usually not be well depicted on nephrographic phase images, because they are incompletely opacified at this stage of the scan. Since the initial 3D reformatting investigations were published in 1994, standard diagnostic renal CT protocols have been modified to address this limitation. A third scan is now employed to image the distended collecting system during the pyelographic phase (typically with a 120-480 second delay) (Olson et al 1995). Incorporating the resulting data into either volume rendering or MSSD images along with the nephrographic phase data, improves collecting system detail in the 3D images at the price of increasing the professional and computer time required to produce the images.

To reliably assess vascular detail using existing CT protocols, an additional spiral CT study is required, because the acquisition of CT angiography (CTA) data requires a faster $(3.5-5.0 \mathrm{cc} / \mathrm{sec})$ injection rate and image 
acquisition earlier in the contrast material injection (typically with a 15 - 25 second delay) (Olson et al 1995). The cost of adding this additional information includes more image processing time, more intravenous contrast material, and more radiation exposure.

\section{Current Developments}

We are currently working with certain refinements to the MSSD techniques used in our previous investigations (Figures $\underline{2}, \underline{3}, \underline{4}$ ). These images are superior to the previously described MSSD images in a number of ways. Notably, they can be co-registered with and displayed superimposed on the source axial images, thus allowing extremely precise depiction of internal anatomic structure and tumor margin at the same time they retain the unambiguous surface depiction which is one of the advantages of MSSD in surgical planning. The methods and techniques used to produce them are more sophisticated than MIP, SSD or MSSD.

CT data (51 slices) was transferred to the Surgical Planning Laboratory (SPL) at the Brigham and Women's Hospital using the local network. The original slices were reduced to 256 x 256 pixels, producing a physical pixel size of $2 \mathrm{~mm}$ and the slice thickness of $4 \mathrm{~mm}$. Several anatomical structures (kidney, lesion, vessels, liver, etc.) were segmented by a semi-automatic method. Later, manual editing was used to improve the results of this automatic segmentation. Both the automatic segmentation and the manual editing were done using MRX (GE Medical Systems, Milwaukee, Wis), a software package that provides a wide range of image processing routines for medical purposes (Cline and Lorensen 1988 and 1990). Next, the 3D models of the structures of interest were generated from the segmented slices using the Surface Model Generator (SMG). SMG is a part of a software package developed at SPL for 3D model generation and viewing. It is based on the Visualization Toolkit (VTK), and uses the Marching Cubes Algorithm for surface generation (Lorensen et al 1987). The viewer allows the user to control the model's position, orientation, color, reflectivity, lighting and point of view (Shenton et al 1995 , Kikinis et al 1996), and to save snapshots of the models for integration into the Anatomy Browser, a Java applet developed at SPL, that provides a convenient framework for integrating various types of information relevant to a case: text (the structure names), 3D images (the surface models), segmented slices and the original CT scan. Both image intensity and depth information are stored in the snapshots, thus making it possible to co-register the 3D model and the axial slices, as well as to employ various advanced display capabilities. For example, surfaces can be made partially or completely transparent, allowing simultaneous display of internal surfaces and structures.

MRX also provides a surface model generator, using an algorithm similar to the one used by VTK (*Lorensen, 1987, Schroeder 1996). A separate set of 3D models was generated using MRX and displayed using the LAVA tool. Next SLICER (a visualization tool developed at SPL) (Hata et al 1997) was used to merge the axial images from the CT dataset with the 3D models. SLICER provides an alternative to the Anatomy Browser framework for combining various types of data. It allows the user to view any cross-section of the scan by specifying it on the 3D model. Appropriate data from the pre-segmented or segmented slices is then mapped onto the 3D model. The processing and display routines described above were performed on Sun computer workstations (Ultra-1; Sun Microsystems, Mountain View, CA).

\section{Future Promise of 3D Imaging}

The technique, indications, advantages, and disadvantages of CTA as compared to standard angiography are beyond the scope of this review (Olson et al 1995). However, it is important to note that although the data from spiral CTA data sets are often displayed as MIP images, (Figure 1) the same data set could be incorporated into a 3D CT image display mode such as MSSD or volume rendering. This would address one of the limitations of 3D imaging discussed above. Nevertheless, if the primary vascular study in a pre-operative workup is to be standard angiography, the additional contrast load required for CTA is difficult to justify, especially for partial nephrectomy candidates, in whom preservation of limited renal function may be important. However, CTA offers certain advantages over conventional angiography (Olson et al 1995), thus it is possible that future hardware and software improvements and the outcome of further clinical trials may allow CT to replace conventional angiography, at least in the pre-operative evaluation of partial nephrectomy candidates. Alternatively, given the capability of advanced spiral CT scanners to perform back-to-back spiral CT scans, it is conceivable that a new protocol could be designed to acquire both CTA data and the standard diagnostic renal 
CT data with the same single bolus contrast media injection. Either of these developments could eliminate the additional contrast media load which currently precludes CTA for pre-operative workup, effectively limiting the quality of vascular anatomy that can be acquired for incorporation in 3D CT images.

To provide the maximum benefit to the patient, the acquisition and display of pyelographic phase data would also have to be of sufficiently high quality to allow adequate pre-operative evaluation of the extrarenal and intrarenal collecting system. Greater clinical experience with CT pyelography will be required before this becomes standard practice. For the purpose of conducting initial studies in this regard, MIP images may be the most useful form of 3D data display. These images could be produced and offer clear comparisons with images from intravenous urography, because MIP images can be produced to resemble standard urographic projections. Ultimately, to avoid having to produce more images than necessary and to reduce the professional time, hardware and software costs to a reasonable level, experience will have to be gained in use of MSSD or volume rendering of CT pyelography images for pre-operative diagnostic evaluation as well as surgical planning.

If future clinical experience validates the utility of CT angiography and pyelography in surgical planning, a spiral CT dataset containing all of the data required for preoperative diagnosis and optimal surgical planning could be acquired in a single hospital visit involving the same spiral CT scan used to diagnose and characterize a renal mass. In order for 3D images to display the internal relationships between tumor, renal parenchyma, intrarenal collecting system, and renal vasculature, advanced MSSD with superimposed axial image displays (as illustrated above) or volume rendering techniques will become a necessity. Only these advanced methods can display such a wealth of data in a small number of compact and intuitively comprehensible images.

When such technology becomes available, the techniques discussed above may prove useful in other types of urologic evaluation and surgical planning. Potential applications to stone disease, for example have recently been illustrated (Sommer et al 1997). Other possible future directions include virtual nephroscopy, ureteroscopy, and cystoscopy (Sommer et al 1997). Because of the large size of the urinary bladder compared to the other structures of interest, cystoscopy is the most feasible at this time, but virtual nephroscopic images have also been obtained (Sommer et al 1997 and Vining et al,1996). Ureteroscopy is more difficult because it entails acquisition of extremely high-resolution CT images. However, for now, the development of all three techniques depends more generally on progress in the field of virtual endoscopy (Jolesz 1997). For any noninvasive virtual endoscopic techniques to have utility, the images will have to be demonstrated to have a high enough sensitivity that negative studies will obviate the need for further investigation. If this becomes possible, virtual endoscopic techniques may prove extremely useful in screening. These studies are less invasive, less costly, and faster than conventional endoscopy. Patients with positive findings on virtual endoscopic studies may still require conventional cystoscopy, ureteroscopic or nephroscopy for biopsy or therapeutic intervention, but patients with negative virtual endoscopy studies could be spared the time, expense, and morbidity of conventional endoscopic procedures. This promises to provide a single, rapid, inexpensive, noninvasive, highly sensitive study for such diverse common presentations as hematuria, and flank pain, which could in theory offer substantial patient benefit, and possibly cost savings, as compared with standard workups.

\section{Figure Legend:}

Figure 1: Spiral CT angiogram. There is a cyst in the lower pole of the left kidney.

Figure 2-4: Spiral CT of the abdomen and pelvis was performed, both before and after intravenous administration of $100 \mathrm{cc}$ of Omnipaque 300, to evaluate gross hematuria in a 63 year old woman. It was acquired with $5 \mathrm{~mm} / \mathrm{sec}$ table feed and $5 \mathrm{~mm}$ collimation, reconstructed in $5 \mathrm{~mm}$ thick sections at $3 \mathrm{~mm}$ increments with a matrix 512 x 512 pixels. The scan revealed a nonenhancing multiseptated cystic mass of intermediate attenuation in the upper pole of the left kidney (arrowheads). A $5 \mathrm{~cm}$ diameter lesion was noted in the right adrenal gland which probably represents a myelolipoma (arrow). At surgery the upper pole renal lesion was found to be adherent to surrounding structures, so a total nephrectomy was performed. Pathology of the resected lesion revealed it to be a complicated cyst. 
a:
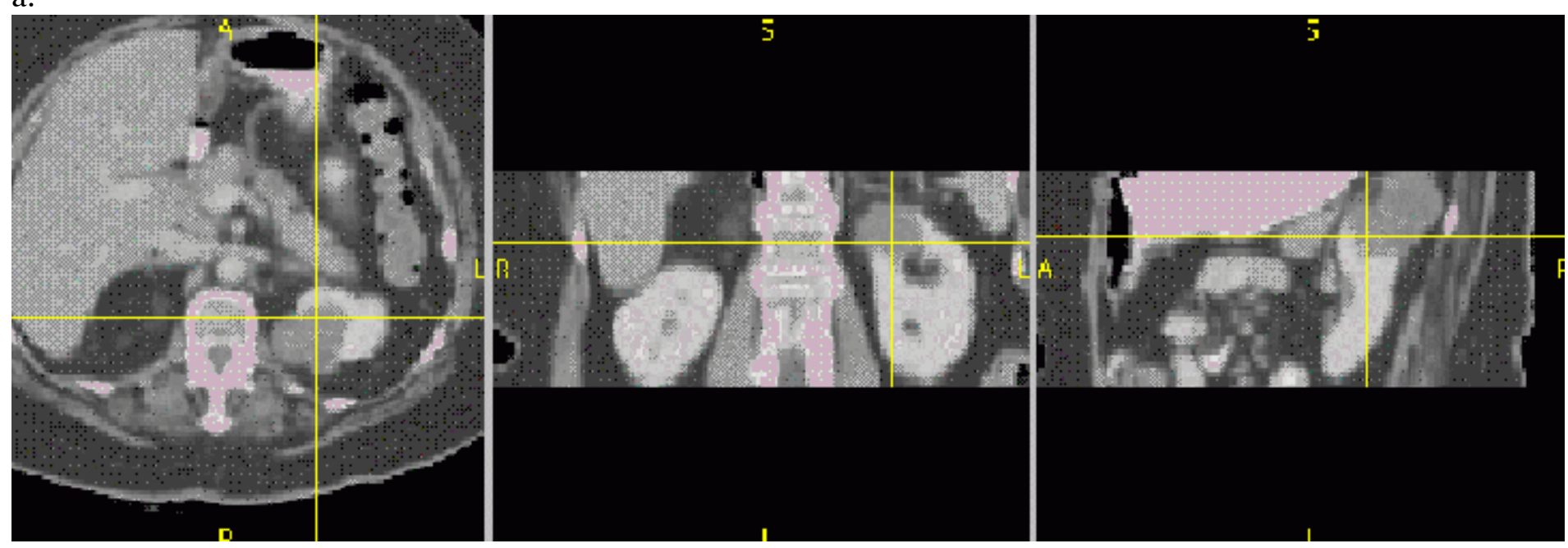

b:
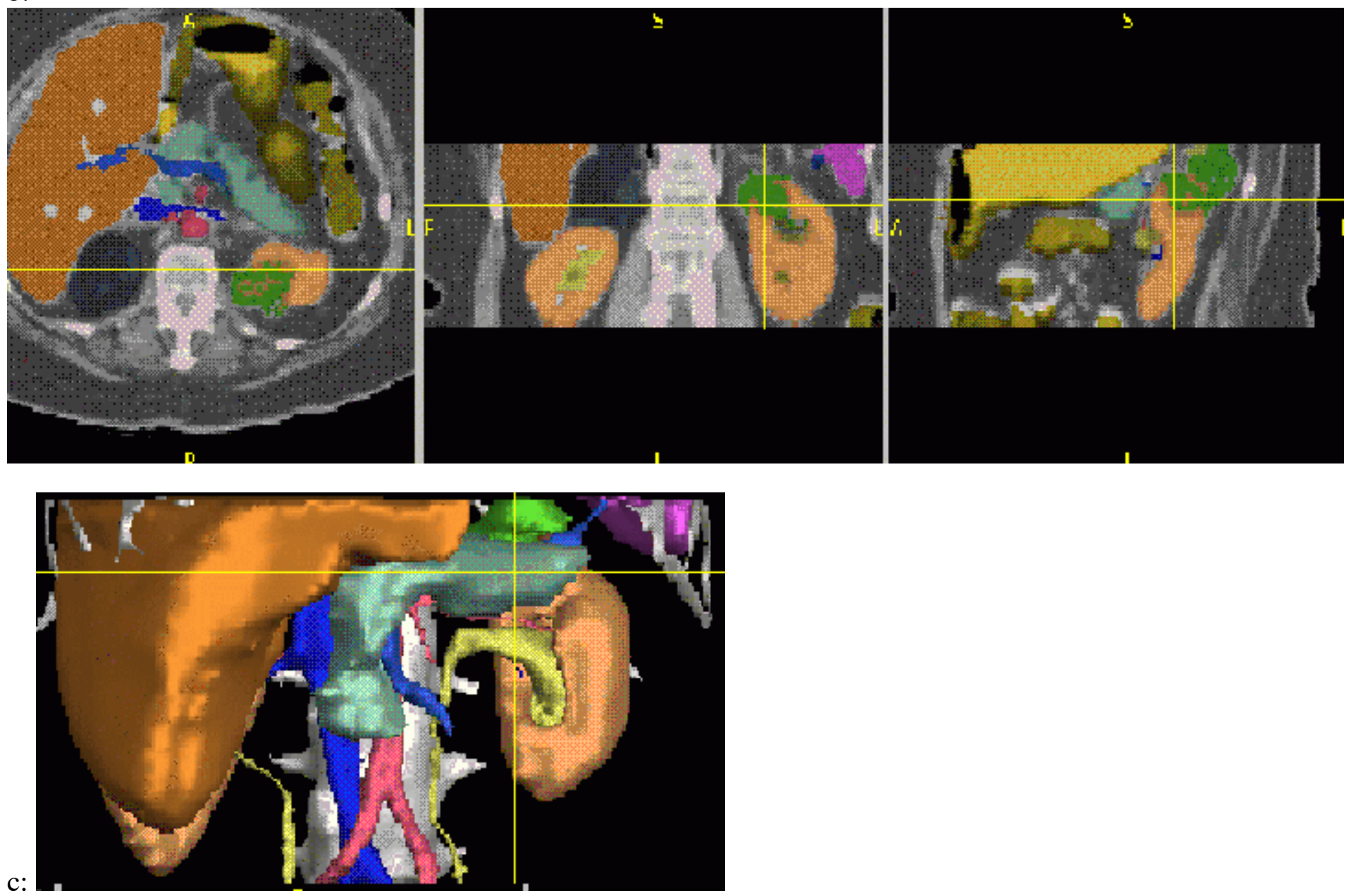

Figure 2: An Anatomy Browser view, demonstrating the relationship between orthogonal image planes and the 3D model displaying liver, pancreas, kidney, renal lesion, spleen, intrarenal collecting system, vessels, bone. a. Corresponding axial (left), coronal (middle) and sagittal (right) image planes reconstructed from the same spiral CT dataset.

b. Anatomical structures color-encoded to highlight the different organs on the grayscale images.

c. 3D model generated by SMG. When the mouse is used to position the crosshairs on one of the orthogonal views, crosshairs are activated on the corresponding image planes, as well as on the 3D model. In real time, the model can be rotated at will. In this snapshot, taken from a frontal point of view, the lesion (green) is partially covered by the pancreatic tail. 


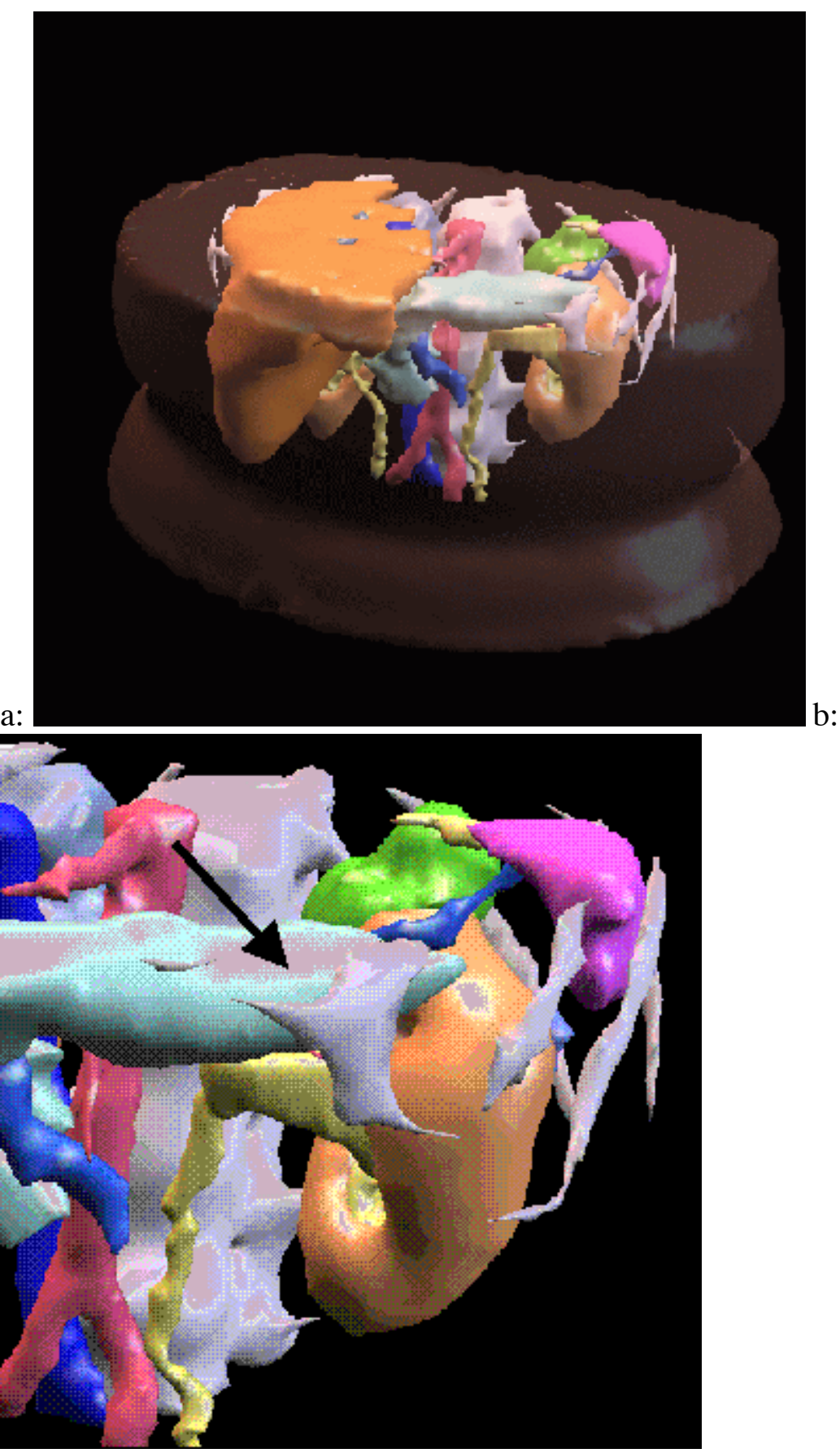



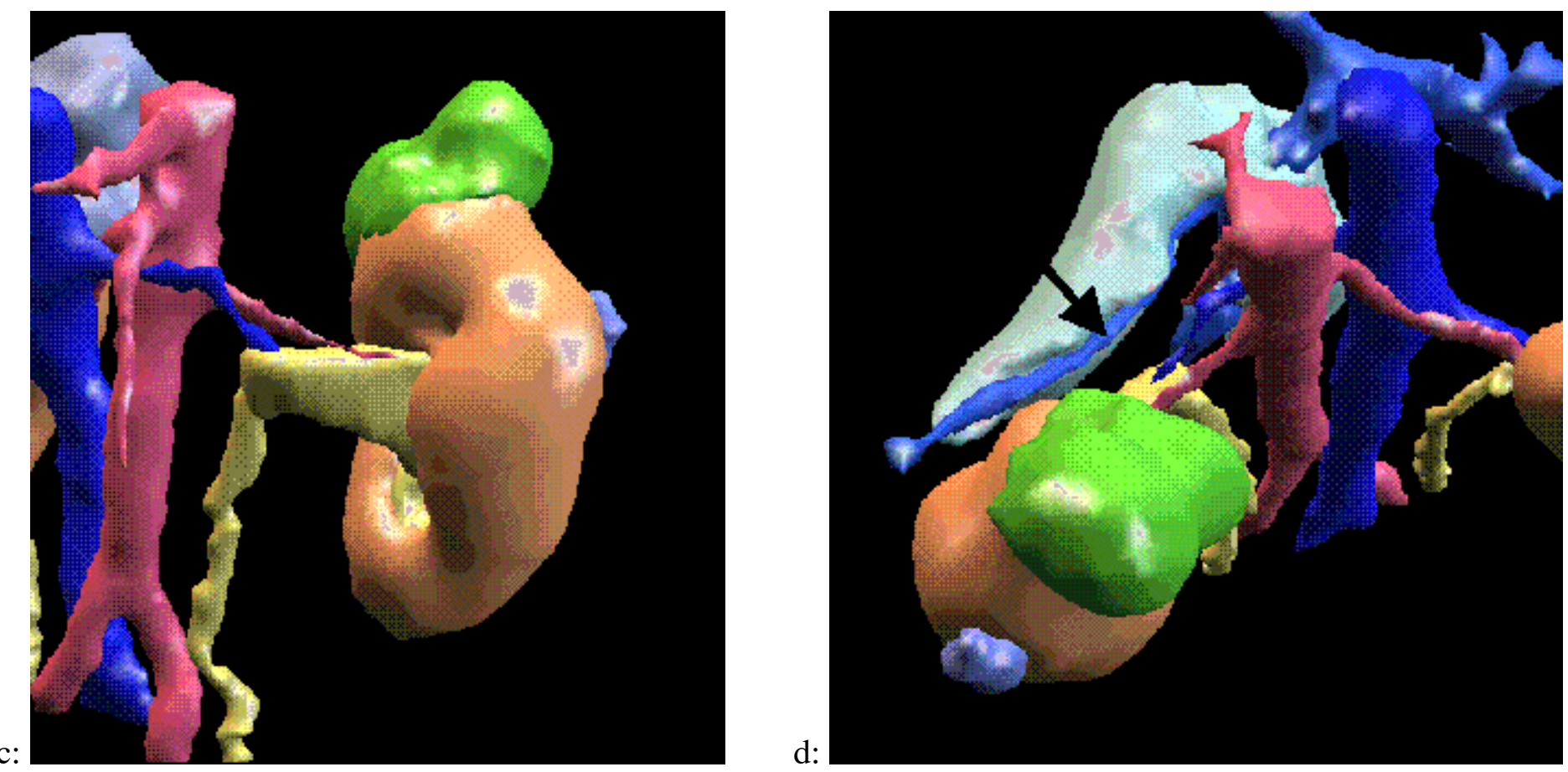

Figure 3: 3D model of the renal lesion and surrounding structures displayed using the LAVA tool.

a. Overview showing transparent skin and opaque liver, pancreas, vessels, intrarenal collecting system, kidney, tumor, spleen, fibrosis and bony structures.

b. Detailed view of the 3D model displaying the relationship of the pancreas (arrow) and other surrounding organs to the left of the renal lesion.

c. The same view of the 3D wall with the surrounding structures removed in order to show the location of the left upper pole renal lesion (green), the vessels, the intrarenal collecting system, and the lateral midpole cyst (purple).

d. The same 3D model viewed from above, demonstrating the relationship of the lesion, the kidney, the splenic vein, the pancreatic tail (arrow), and other structures.

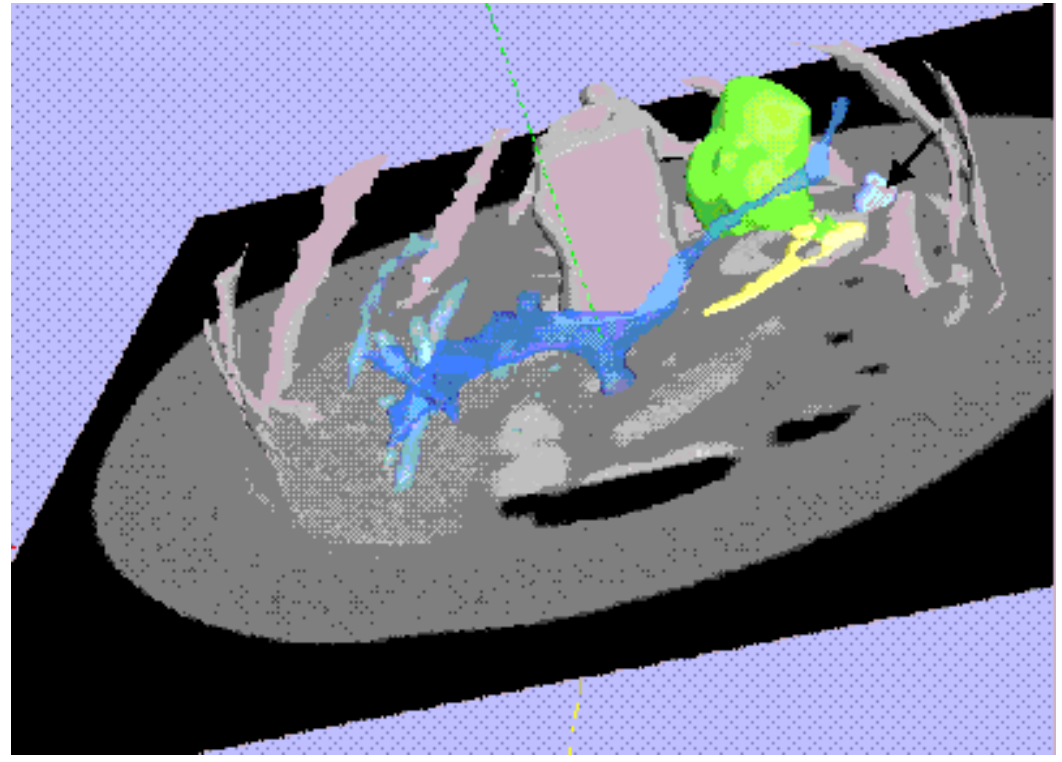

Figure 4: Axial source image co-registered with the 3D model and integrated into a comprehensive display using SLICER. Bone and skin were used as references to merge axial grayscale images retrieved from the CT dataset with the 3D model. The portal and the splenic veins, the intrarenal collecting system, and their relationship to the lesion and to the cyst (arrow) are displayed. The kidney has been removed from the 3D model in order to improve visibility of the kidney (arrow) on the corresponding axial CT image. 


\section{References}

1. Chernoff DM, Silverman SG, Kikinis R: Three-dimensional imaging and display of renal tumors using helical CT. Urology 43:125, 1994.

2. Cline HE, Lorensen WE, Ludke S, Crawford CR, Teeter BC: Two algorithms for the three-dimensional reconstruction of tomograms Med Phys. 15:320-327, 1988.

3. Cline HE, Lorensen WE, Kikinis R, Jolesz FA: Three-dimensional segmentation of MR images of the head using probability and connectivity J Computed Assist Tomogr . 14: 1037-1045, 1990.

4. Fishman EK, Magid D, Ney DR: Three-dimensional imaging. Radiology 181:321-337, 1991.

5. Hata N, Kettenback J, Wells WM, Westin CF, Nakajima S, Kikinis R, Jolesz FA: Registration of pre-operative and intra-operative images for MR-guided therapy. J Magn Reson Imag (in press)

6. Jolesz FA, Lorenson WE, Shinmoto H, Atsumi H, Nakajima S, Kavanagh P, Saiviroonporn P, Seltzer SE, Silverman SG, Phillips MD, Kikinis R: Interactive virtual endoscopy. Am J Roentgenol (in press).

7. Kalendar WA, Seissler W, Klotz E, Vock P: Volumetric CT with single breath-hold technique, continuous transport and continuous scanner rotation. Radiology 176:18-183, 1990.

8. Kikinis R, Shenton ME, Iosifescu DV, McCarley RW, Saiviroonporn P, Hokama HH: A digital brain atlas for surgical planning, model driven segmentation and teaching. IEEE Transactions on visualization and computer graphics, Vol.2, (3) 1996.

9. Lorensen WE, Cline HE: Marching Cubes: A high resolution 3D surface construction algorithm. Computer Graphics 21(4): 163-139, 1987.

10. Madjar S, Moskovitz B, Halachmi S, Nativ O: Three-dimensional imaging using spiral computed tomography prior to tumor enucleation in a patient with a solitary kidney. Urologia Internationalis 56: 112-113, 1996.

11. Olson MC, Posniak HV: Urologic applications of multiplanar and three-dimensional computed tomography. Techniques in Urology 1(3): 141-149, 1995.

12. Rubin DR, Silverman SG: Helical (spiral) CT of the retroperitoneum. Radiol Clin North Am 33:903, 1995.

13. Schroeder W, Martin K, Lorensen B: The Visualization Toolkit. An object-oriented approach to 3D graphics. Prentice Hall, New Jersey, 1996.

14. Shenton ME, Kikinis R, McCarley, Saiviroonporn P, Hokama HH, Robatino A: Harvard Brain Atlas: A teaching and visualization tool. Proceedings: Biomedical Visualization , p10 -17, 1995.

15. Silverman SG, Bloom DA, Seltzer SE: The radiological evaluation of renal masses-approach, analysis, and new technologies. AUA Update Series, Lesson 1, Volume XIII, 1994.

16. Silverman SG, Lee BY, Seltzer SE, Bloom DA, Corless CL, Adams DF: Small renal masses: correlation of spiral CT features with pathologic findings. Am J Roentgenol 163:597-605, 1994.

17. Silverman SG, Pearson GDN, Seltzer SE, Polger M, Tempany CMC, Adams DF, Brown DL, Judy PF: Small $(? 3 \mathrm{~cm})$ hyperechoic renal masses: comparison of helical and conventional CT for diagnosing angiomyolipoma. Am J Roentgenol 167:877-881, 1996.

18. Sommer FG, Okott EW, Ch'en I, Beaulieu CF: Volume rendering of CT data: applications to the genitourinary tract. Am J Roentgenol 168:1223-1226, 1997. 
19. Vining DJ, Zagoria RJ, Liu K, Stelts D: CT cystoscopy: an innovation in bladder imaging. Am J Roentgenol 166:409, 1996. 